\title{
Estudio de la capacidad de biomasa forrajera accesible del \\ CIPTT de Tullpacancha - en condiciones climáticas normales \\ - para determinar la densidad poblacional de vicuñas
}

Study of the accessible capacity of biomass forage of the CIPTT of Tullpacancha

- in normal climatic conditions - to determine the population density of Vicuñas

Jaime Deza Rivasplata ${ }^{1}$, Luis Chacón ${ }^{2}$ y otros

http://dx.doi.org/10.21503/CienciayDesarrollo.2006.v6.03

RESUMEN

Se desea conocer la variedad de pastos, calidad, cantidad y distribución de ellos, para determinar la soportabilidad en relación al número de vicuñas que el Centro de Investigación, Producción y Transferencia Tecnológica CIPTT de Tullpacancha puede criar. Se obtiene la soportabilidad de 3 vicuñas por hectárea, a diferencia del promedio nacional que es de 5 hectáreas para una vicuña.

\section{ABSTRACT}

It is desired to know the variety of grass, quality, amount and distribution of them, to determine the supportability in relation to the number of vicuñas that Research, Production and technological Transference Center CIPTT of Tullpancha can raise. The supportability of 3 vicuñas is obtained by hectare, unlike of the national average that is of 3 hectares for one vicuña.

\section{INTRODUCCIÓN}

EI CIPTT de la Universidad Alas Peruanas está ubicado en el distrito de Churcampa, provincia Churcampa, departamento Huancavelica. Comprende un área de 1000 hectáreas, (3 900 a 4200 m.s.n.m.), con una temperatura promedio anual de 8 a $10^{\circ} \mathrm{C}$ y un promedio de precitación anual de 900 a $1000 \mathrm{~mm}$.

La configuración topográfica está definida por áreas onduladas y colinas, típicas de las laderas que encierran estos valles y el escenario edáfico está conformado por suelos relativamente profundos (que van de 20 a 60 centímetros de espesor), de textura media, con buen drenaje. De acuerdo a los análisis de suelos efectuados por el laboratorio de suelos de la Universidad Nacional
Agraria La Molina, Lima, Perú, el suelo es de alta a mediana fertilidad, con alto porcentaje de materia orgánica, con suelos oscuros de estructura granular, con pH ácido a muy ácido (6,5 a 6), con contenidos de nitrógeno mineral de 102 a 156 kg/ ha/año, contenidos altos a medianos de fósforo y contenidos altos de potasio. No existen problemas de salinidad.

Con respecto a los tipos de pastizal, se encuentran cuatro tipos: Pajonal, Césped de Puna, Bofedal y Canllar. En paneos de observación, se ha registrado que 62 especies forrajeras son consumidas por las vicuñas.

\section{MATERIAL Y MÉTODO}

El estudio se ha realizado durante los me-

1. Jaime Deza Rivasplata, Antropólogo. Director del Centro de Investigación de la Universidad Alas Peruanas.

2. Luis Chacón Navarro, Ingeniero zootenista, Director del CIPTT Tullpacancha, Danny Sánchez Centty, Médico veterinario, Lissie Palacios Urtado, Meyling Cheng Long, Escuela de Medicina Veterinaria UAP 
ses de abril a noviembre del año 2006, que com- Nacional Mayor de San Marcos.

prende a una época sin lluvias.

Identificadas las variedades, por observa-

El registro de la biomasa forrajera se ha ción directa se registraron los de mayor preferenrealiado en paneos (cuadrículas de $10 \times 10 \mathrm{~m}$ ) tocia por las vicuñas (que además resultaron ser de mados al azar considerando dos cuadrículas por mayor valor nutrituvo y de mayor concentración en hectárea. el área).

La identificación en el campo de los tipos Los análisis de laboratorio se realizaron de pastos se ha realizado por observación directa, seleccionándose las plantas representativas para luego ser identificadas en el gabinete respectivo en los laboratorios de Evaluación Nutricional de Alimentos de la Universidad Nacional Agraria La Molina, Lima, Perú del Museo de Historia Natural de la Universidad

\section{RESULTADOS}

Considerando las variables de: mayor preferencia por las vicuñas y de valor nutricional, se identificaron once variedades de pastos.

Tabla 1 Pastos de mayor aceptación y valor nutricional

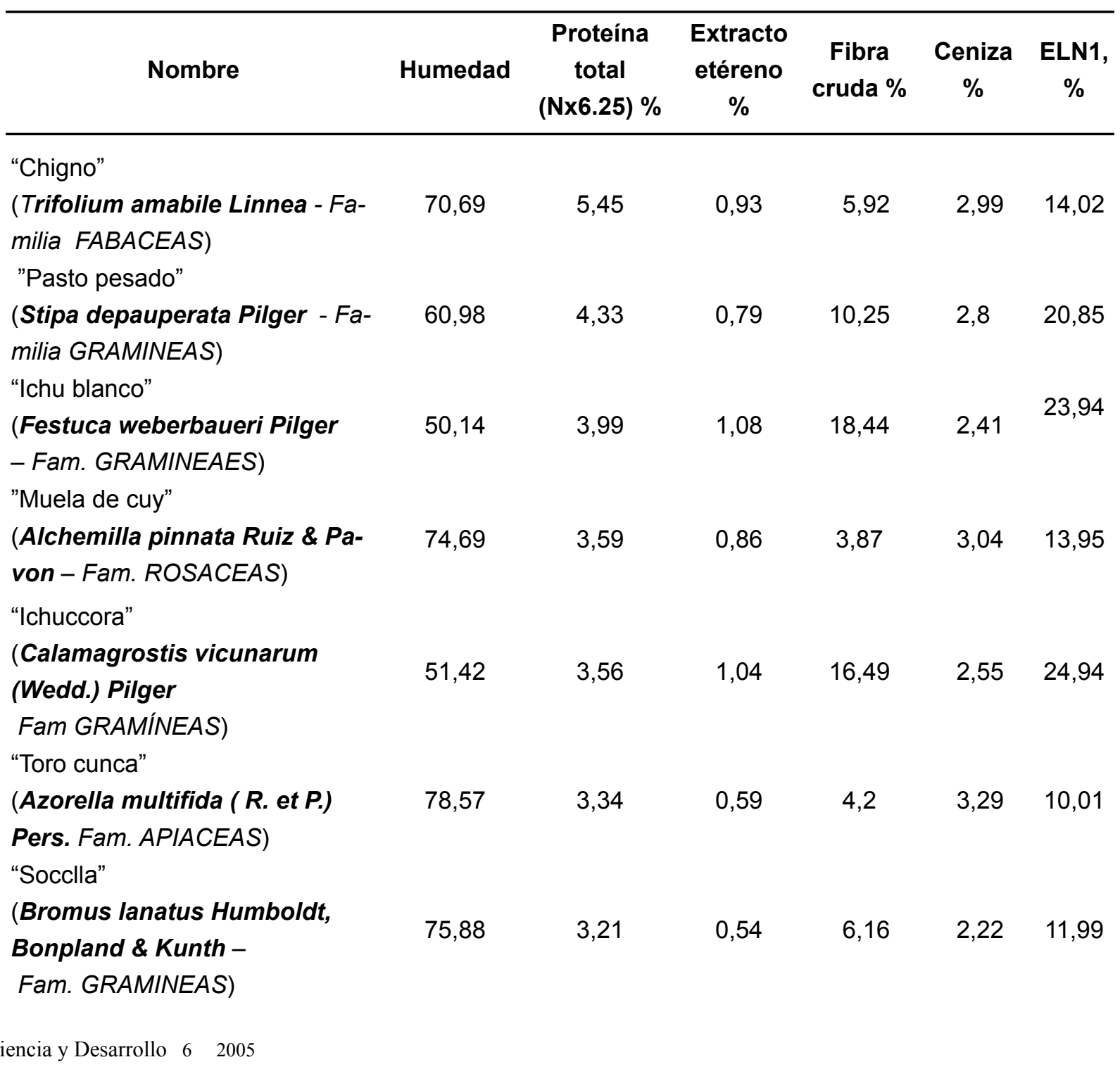


"Hierba colorada" o "Puka locco"

\begin{tabular}{|c|c|c|c|c|c|c|}
\hline $\begin{array}{l}\text { (Rumex acetosella Linneo } \\
\text { - Fam. POLYGONACEAS) }\end{array}$ & 82,19 & 3,17 & 0,57 & 3,4 & 1,16 & 9,51 \\
\hline $\begin{array}{l}\text { Grama de Puquio (*) } \\
\text { "Yanapuqio" }\end{array}$ & 74,36 & 2,75 & 0,84 & 7,56 & 1,3 & 13,19 \\
\hline $\begin{array}{l}\text { (Carex ecuadorica Kukenth } \\
\text { - Fam. CYPERACEAS) }\end{array}$ & 84,91 & 2,27 & 0,66 & 2,48 & 1,7 & 7,98 \\
\hline “Ccanccagua” & & & & & & \\
\hline $\begin{array}{l}\text { (Stylitis andicola Amstutz } \\
\text { - ISOETACEAS) }\end{array}$ & 85,87 & 0,97 & 0,28 & 2,33 & 1 & 9,55 \\
\hline
\end{tabular}

(*) Sin identificación

Fuente: Laboratorio de Evaluación Nutricional de Alimentos, Universidad Nacional Agraria La Molina.

Distribución porcentual de las variedades forrajeras y Biomasa Forrajera Accesible.

Considerando los promedios obtenidos en los paneos de observación las 11 variedades más frecuentes están distribuidas de la manera siguiente (Tabla 2).
De las once (11) variedades forrajeras la: las "Muela de cuy" (Alchemilla pinnata Ruiz \& Pavon - Fam. ROSACEAE), "Socclla" (Bromus lanatus Humboldt, Bonpland \& Kunth - Fam. GRAMINEAE), "Puka locco (Rumex acetosella linneo - Fam. POLYGONACEAE) son las de mayor producción por corte, superando el $60 \%$ de la producción total del área foliar comestible (2 164 TM por corte) distribuidas en el $30 \%$ del área total (300 hectáreas).

Tabla 2 Distribución porcentual de las variedades forrajeras en el CIPTT.

\begin{tabular}{llc}
\hline \multicolumn{1}{c}{ Nombre común } & \multicolumn{1}{c}{ Nombre científico } & $\begin{array}{c}\% \text { del área } \\
\text { total }\end{array}$ \\
\hline $\begin{array}{l}\text { Muela de cuy } \\
\text { Socclla }\end{array}$ & $\begin{array}{l}\text { Alchemilla pinnata Ruiz \& Pavon - Fam. ROSACEAS } \\
\text { Bromus lanatus Humboldt, Bonpland \& Kunth - Fam. } \\
\text { GRAMINEAS }\end{array}$ & 11,0 \\
Puka locco & Rumex acetosella linneo - Fam. POLYGONACEAE & 10,0 \\
Chigno & Trifolium amabile Linnea - Fam. FABACEAS & 9,0 \\
Icchuccora & Calamagrostis vicunarum (Wedd.) Pilger - Fam & 5,0 \\
Icchu blanco & GRAMíNEAS & 8,0 \\
Pasto pesado & Festuca weberbaueri Pilger - Fam. GRAMINEAE & 4,0 \\
Toro ccunca $\left(^{*}\right)$ & Stipa depauperata Pilger - Familia GRAMINEAS & 1,0 \\
Gramíneas comestibles & Azorella multifida (R. et. P.) Pers. - Fam. APIACEAS & 1,0 \\
Gramíneas no comestibles & & 13,0 \\
Plantas de oconales: & & 16,0 \\
Ccanccagua & & \\
Yana puquio & Stylitis andicola Amstutz - Fam. ISOETACEAS \\
Grama puquio $\left(^{*}\right)$ & (Carex ecuadorica Kukenth - Fam. CYPERACEAS) & 3,92 \\
Otras plantas & & 2,10 \\
Rocas, pedregal, & & 1,68 \\
TOTAL: & & 6,3 \\
\hline
\end{tabular}

(*) Sin identificación 
Tabla 3 Biomasa forrajera accesible

\begin{tabular}{|c|c|c|c|c|c|}
\hline $\begin{array}{l}\text { Nombre } \\
\text { Común }\end{array}$ & Nombre Científico & $\begin{array}{l}\text { Rendimiento: } \\
\text { Gramos x M2. }\end{array}$ & $\begin{array}{l}\text { Área Total. } \\
\text { Hectáreas }\end{array}$ & $\begin{array}{l}\text { B.F.A. } \\
\mathrm{kgr} / \mathrm{Ha}\end{array}$ & $\begin{array}{l}\text { Produc- } \\
\text { ción total. } \\
\text { TM (B.F.A.) }\end{array}$ \\
\hline Muela de cuy & $\begin{array}{l}\text { (Alchemilla pinnata Ruiz \& } \\
\text { Pavon - Fam. ROSACEAS) }\end{array}$ & 560 & 110,0 & 5600 & 616,0 \\
\hline Socclla & $\begin{array}{l}\text { (Bromus lanatus Humboldt, } \\
\text { Bonpland \& Kunth - Fam. } \\
\text { GRAMINEAS) }\end{array}$ & 720 & 100,0 & 7200 & 720,0 \\
\hline Puka locco & $\begin{array}{l}\text { Rumex acetosella Linneo } \\
\text { - Fam. POLYGONACEAE) }\end{array}$ & 920 & 90,0 & 9200 & 828,0 \\
\hline Chigno & $\begin{array}{l}\text { Trifolium amabile Linnea - } \\
\text { Fam. FABACEAS }\end{array}$ & 250 & 50,0 & 2500 & 125,0 \\
\hline Icchuccora & $\begin{array}{l}\text { Calamagrostis vicuna- } \\
\text { rum (Wedd.) Pilger - Fam } \\
\text { GRAMIINEAS }\end{array}$ & 670 & 80,0 & 6700 & 536,0 \\
\hline Icchu blanco & $\begin{array}{l}\text { (Festuca weberbaueri Pil- } \\
\text { ger-Fam. GRAMINEAES) }\end{array}$ & 320 & 40,0 & 3200 & 128,0 \\
\hline Pasto pesado & $\begin{array}{l}\text { (Stipa depauperata Pilger - } \\
\text { Familia GRAMINEAS) }\end{array}$ & 450 & 10,0 & 4500 & 45,0 \\
\hline Toro ccunca & $\begin{array}{l}\text { Azorella multifida (R. et. P.) } \\
\text { Pers. - Fam. APIACEAS }\end{array}$ & 830 & 10,0 & 8300 & 83,0 \\
\hline \multicolumn{6}{|l|}{$\begin{array}{l}\text { Plantas de } \\
\text { oconales }\end{array}$} \\
\hline Ccanccagua & $\begin{array}{l}\text { (Stylitis andicola Amstutz } \\
\text { - ISOETACEAS) }\end{array}$ & 880 & 39,2 & 8800 & 344,96 \\
\hline \multirow[t]{2}{*}{ Yana puquio } & (Carex ecuadorica & & & & \\
\hline & $\begin{array}{l}\text { Kunkenth - Fam. YPERA- } \\
\text { CEAE) }\end{array}$ & 450 & 21,0 & 4500 & 94,5 \\
\hline Grama puquio & & 560 & 16,8 & 5600 & 94,08 \\
\hline $\begin{array}{l}\text { Otras plantas } \\
\text { del oconal }\end{array}$ & & & 63,0 & & \\
\hline $\begin{array}{l}\text { Gramíneas } \\
\text { comestibles }\end{array}$ & & & 130,0 & & \\
\hline $\begin{array}{l}\text { Gramíneas no } \\
\text { comestibles }\end{array}$ & & & 160,0 & & \\
\hline $\begin{array}{l}\text { Rocas, pe- } \\
\text { dregales }\end{array}$ & & & 80,0 & & \\
\hline TOTAL & & & 1000,00 & & 3614,54 \\
\hline
\end{tabular}

Registros tomados en los meses de octubre de 2005 y 2006. 


\section{CONCLUSIÓN}

Se observa una biomasa forrajera accesible (B.F.A.) en el CIPTT anual, (con proyecciones conservadoras), de 3614 TM de pasto nativo y de alto valor nutritivo (tomando como punto de comparación a la alfalfa (Medicago sativa) se deduce la existencia de una cantidad de 9900 kilogramos de pasto óptimo para consumo diario (considerando un corte anual).
Si tenemos en cuenta que cada vicuña consume diariamente 1300 gramos de forraje (se obtiene un promedio de consumo total de 4850 $\mathrm{kg}$ ) y que el otros $50 \%(4850 \mathrm{~kg}$ ) correspondería a pérdidas de forrajes por diversas causas, tendríamos alimento suficiente para la crianza en semi cautiverio de 3800 cabezas en condiciones normales.

Tabla 4 Variedades registradas en el CIPTT Tullpacancha

Variedades forrajeras identificadas

\begin{tabular}{|c|c|c|}
\hline Familia & Especie & Nombre común \\
\hline 1. FABACEAS & Trifolium amabile H.B.K & Chigno \\
\hline 2. GRAMÍNEASS & Tipa depauperata Pilger & Pasto pesado \\
\hline 3. GRAMINEAES & Festuca weberbaueri Pilger & Ichu blanco \\
\hline 4. ROSACEAE & Alchemilla pinnata Ruiz \& Pavon & Muela de cuy \\
\hline 5. GRAMÍNEAS & Calamagrostis vicunarum (Wedd.) Pilger & Icchuccora \\
\hline 6. APIACEAS & Azorella multifida (R. et P.) Pers. & Toro ccunca \\
\hline 7. GRAMÍNEAS & $\begin{array}{l}\text { Bromus lanatus Humboldt, Bonpland \& } \\
\text { Kunth }\end{array}$ & Socclla \\
\hline 8. POLYGONACEAS & Rumex acetosella Linneo & Puka locco, hierba colorada \\
\hline 9. Sin identificar & Idem. & Grama puquio \\
\hline 10. CYPERACEAS & Carex ecuadorica Kunkenth & Yanapuqio \\
\hline 11. ISOETACEAS & Stylitis andicola Amstutz & Ccanccagua \\
\hline 12. ONAGRACEAS & Oenothera multicaulis Ruíz \& Pavon & Chupa sangre \\
\hline 13. GERANIACEAS & Geranium patagonicum Hooker $f$. & \\
\hline 14. GRAMINEACEAS & Stipa depauperata Pilger & \\
\hline 15. CARYOPHYLLACEAS & Drymaria sp. & \\
\hline 16. GRAMINEAS & Bromus catharticus Vahl & \\
\hline 17. APIACEAS & Lilaeopsis andina (Hill.) Perez-Moreau. & \\
\hline 18. ASTERACEAS & $\begin{array}{l}\text { Hypochoeris meyeniana (Walper) Grise- } \\
\text { bach }\end{array}$ & \\
\hline 19. CYPERACEAS & Eleocharis albibracteata Nees \& Meyen & Pelo de chancho \\
\hline 20. ROSACEAS & Alchemilla diplophylla Diels & \\
\hline 21. SANTALACEAS & $\begin{array}{l}\text { Quinchamalium procumbens Ruíz y } \\
\text { Pavon }\end{array}$ & \\
\hline 22. VALERIANACEAS & Phillactis rigida (R.\&P.) Pers. & \\
\hline 23. ONAGRACEAS & Oenothera multicaulis $R . \& P$ & \\
\hline 24. PLANTAGINACEAS & Plantago sericea $R . \& P$ & \\
\hline 25. BRASICACEAS & Lepidium chichicara Desvaux & \\
\hline 26. ASTERACEAS & Paranephelius bullatus A. Gray & Achiccora \\
\hline
\end{tabular}




\begin{tabular}{|c|c|c|}
\hline $\begin{array}{l}\text { 27. GRAMINEAS (POA- } \\
\text { CEAS) }\end{array}$ & Calamagrostis rigida (H.B.K) Trinius & Ichu blanco \\
\hline 28. ASTERACEAS & $\begin{array}{l}\text { Hypochoeris taraxacoides (Walper) B. } \\
\& H\end{array}$ & \\
\hline 29. ASTERACEAS & Werneria villosa A. Gray & \\
\hline 30. PORTULACACEAS & $\begin{array}{l}\text { Calandrinia acaulis Humb., Bonpl. \& } \\
\text { Kunth }\end{array}$ & Rabo de chancho \\
\hline 31. VALERIANACEAS & Phyllactis sp. af. Ph. Rigida & \\
\hline 32. GRAMINEAS & Agrostis breviculmis Hitchcok & \\
\hline 33. GRAMINEAS & Muhlenbergia peruviana (Beauv.) Steud. & Icchucha roca \\
\hline 34. GRAMINEAS & Agrostis haenkeana Hichc. & Icchu ccoracha \\
\hline 35. JUNCACEAS & Luzula racemosa Desvaux & \\
\hline 36. GRAMINEAS & Muhlenbergia fastigiata Henrad & Almahadilla \\
\hline 37. GRAMÍNEAS & Calamagrostis amoema (Pilger) Pilger & Icchu suave \\
\hline 38. GRAMINEAS & Poa gymnantha Pilger & \\
\hline 39. JUNCACEAS & Oxychloe andina Philippi & Escobilla \\
\hline 40. RANUNCULACEAS & Ranunculus praemorsus H. B. K & Cintilla amarilla \\
\hline 41. ONAGRACEAS & Oenothera multicaulis $R$. et $P$ & $\begin{array}{l}\text { Jaguar socco, Chupa } \\
\text { sangre }\end{array}$ \\
\hline 42. CARIOFILACEAS & Drymaria sp. & Cojín pasto \\
\hline 43. GRAMINEAS & Muhlenbergia lugularis (Hack.) Hitchc.- & \\
\hline 44. JUNCACEAS & Luzula peruviana Desvaux & \\
\hline 45. IRIDACEAS & Sisyriunchium chilense Hook. & \\
\hline 46. JUNCACEAS & Juncus sp & \\
\hline 47. IRIDACEAS & Sisyrinchium junceum Meyen & \\
\hline 48. GRAMINEAS & $\begin{array}{l}\text { Calamagrostis heterophylla (Wedd.) } \\
\text { Pilger }\end{array}$ & \\
\hline 49. GRAMINEAS & Festuca inarticulata Pilg. & \\
\hline 50. GRAMINEAS & Hordeum muticum Presl & \\
\hline $\begin{array}{l}\text { 51.LEGUMINOSAS (FABA- } \\
\text { CEAS) }\end{array}$ & Trifolium amabile Linneo & \\
\hline 52. GRAMINEAS & Agrostis glomerata (Presl) Kunth & \\
\hline 53. & Trisetum spicatum (Linneo) Richter & \\
\hline 54. GRAMINEAS & Festuca tectoria St-Yves & \\
\hline 55. GRAMÍNEAS & Agrostis tolucensis H.B.K & \\
\hline 56. GRAMÌNEAS & Festuca Casapaltensis J. Ball & \\
\hline 57. GRAMÍNEAS & Festuca setifolia Stendel & \\
\hline 58. GRAMÍNEAS & $\begin{array}{l}\text { Elymus af. E. cordilleranus Davidse et } \\
\text { Pohl }\end{array}$ & \\
\hline 59. GRAMÍNEAS & Juncus imbricatus La Harpe & \\
\hline 60. GRAMÍNEAS & Calamagrostis fuscata (Presl) Steudel & \\
\hline 61. GRAMÍNEAS & Calamagrostis sp. & \\
\hline
\end{tabular}




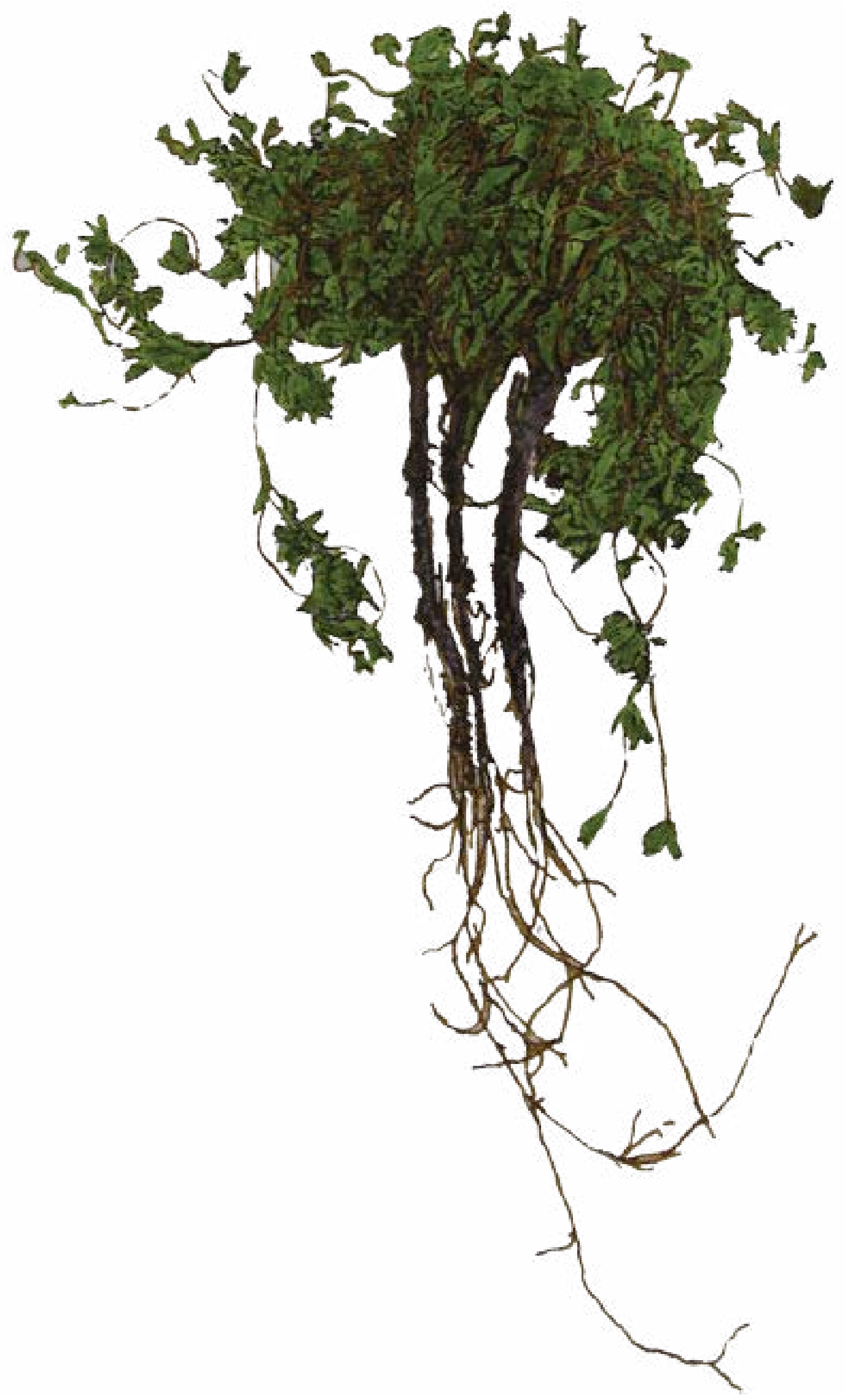

Figura 1. Fabaceas Trifolium amabile H.B.K. Chigno 


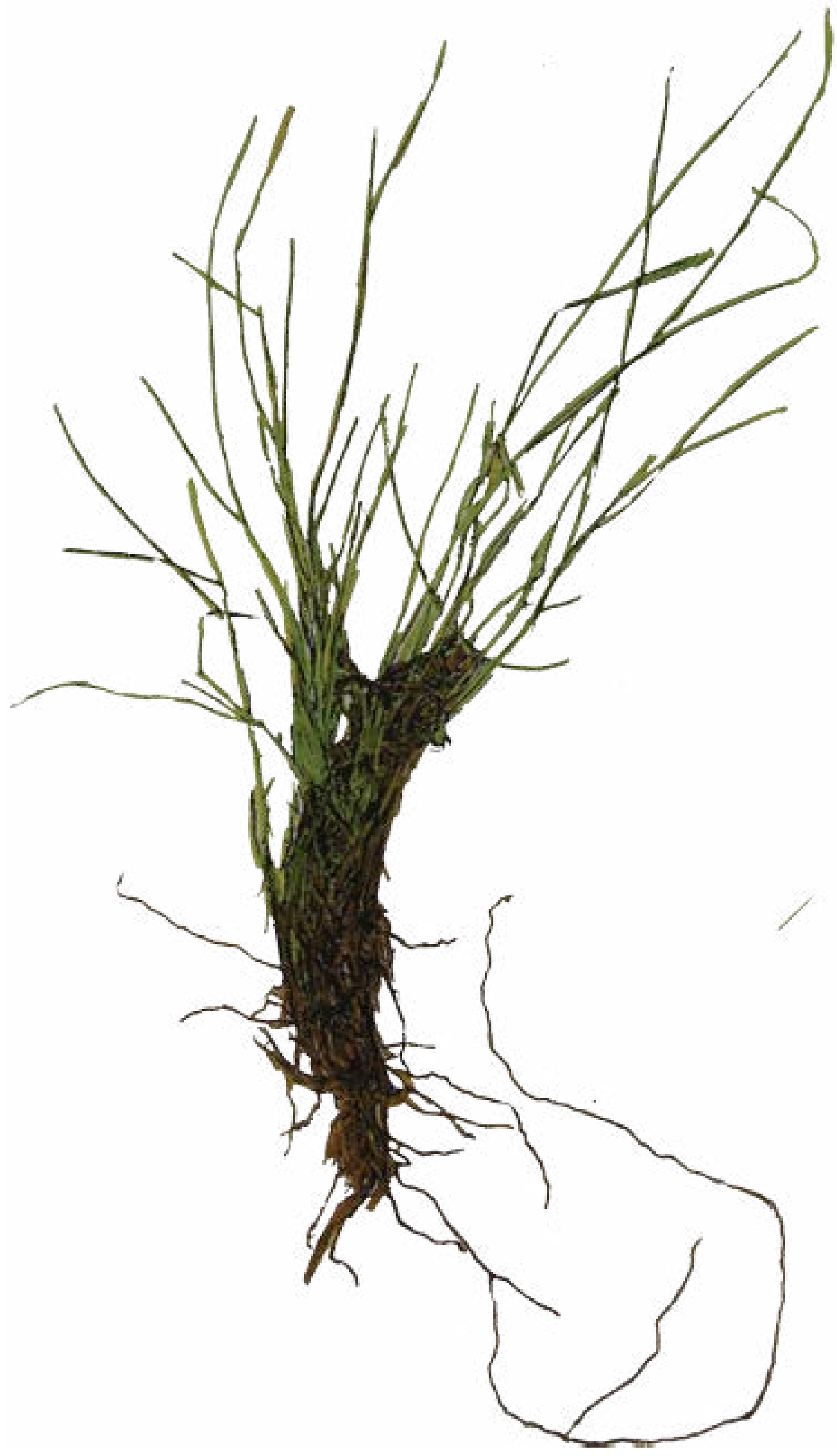

Figura 2. Gramíneas Stipa depauperata Pilger Pasto pesado 


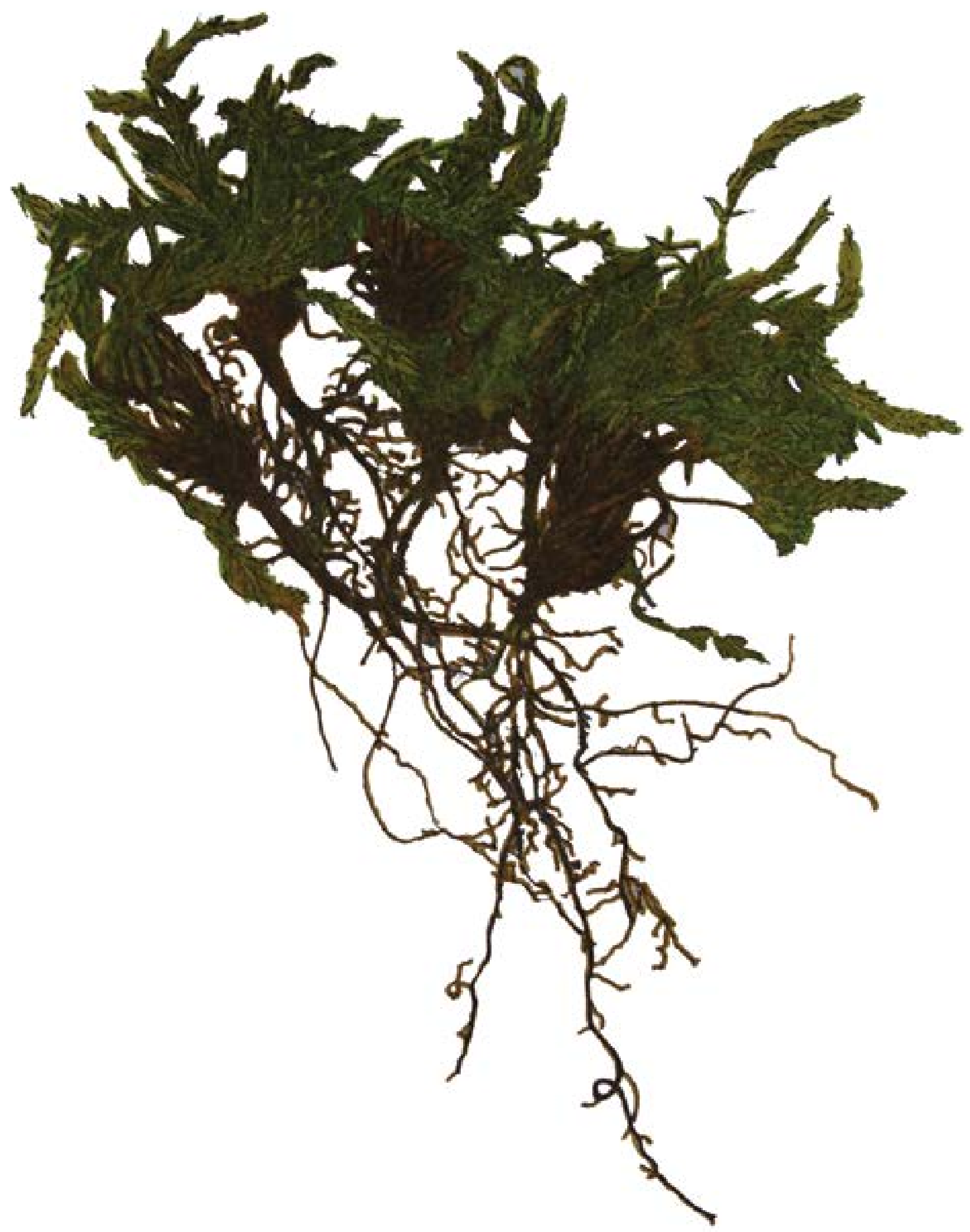

Figura 3. Rosaceas Alchemilla pinnata Ruiz \& Pavon Muela de cuy 


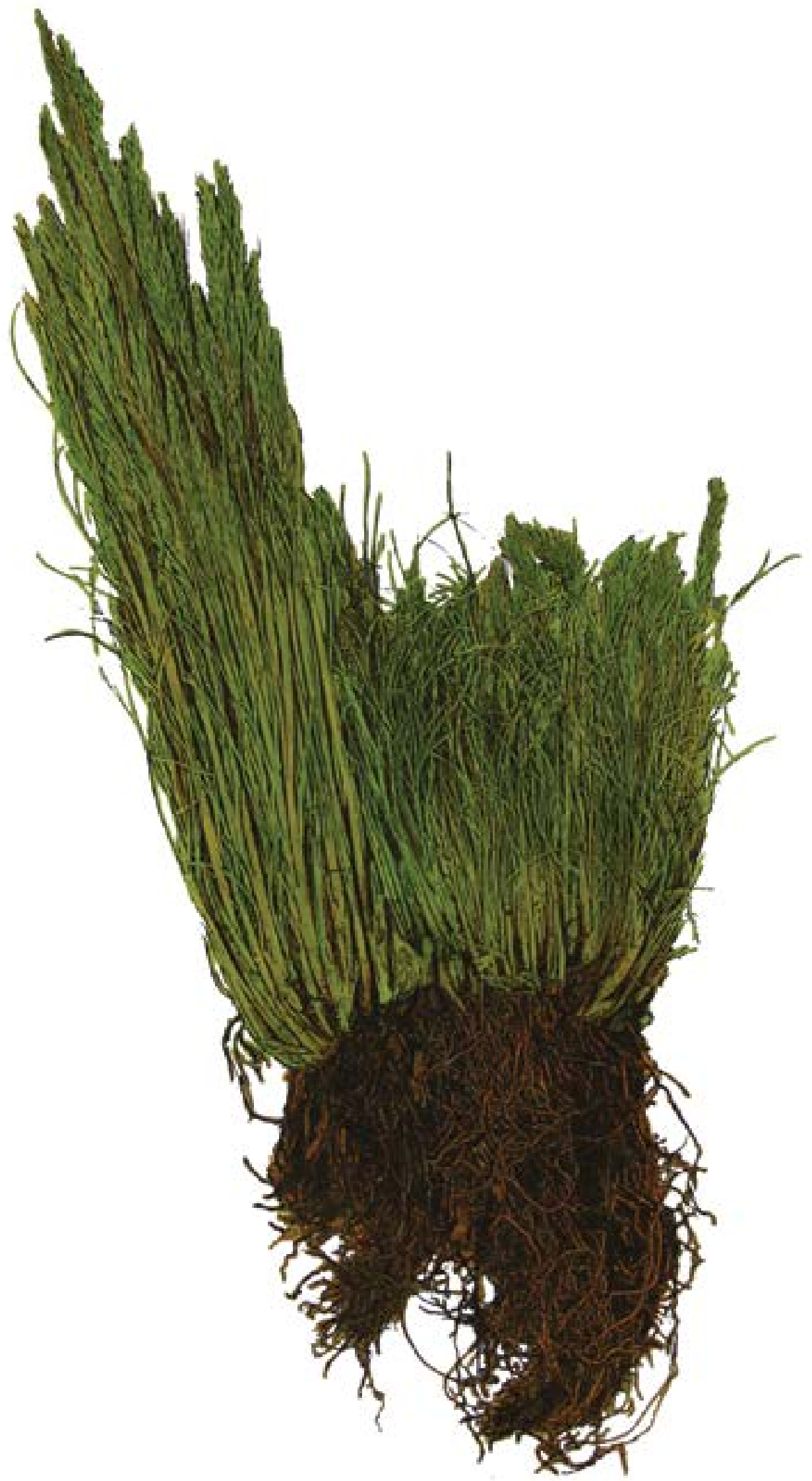

Figura 4. Gramíneas Calamagrostis vicunarum (Wedd.) Pilger Icchuccora 


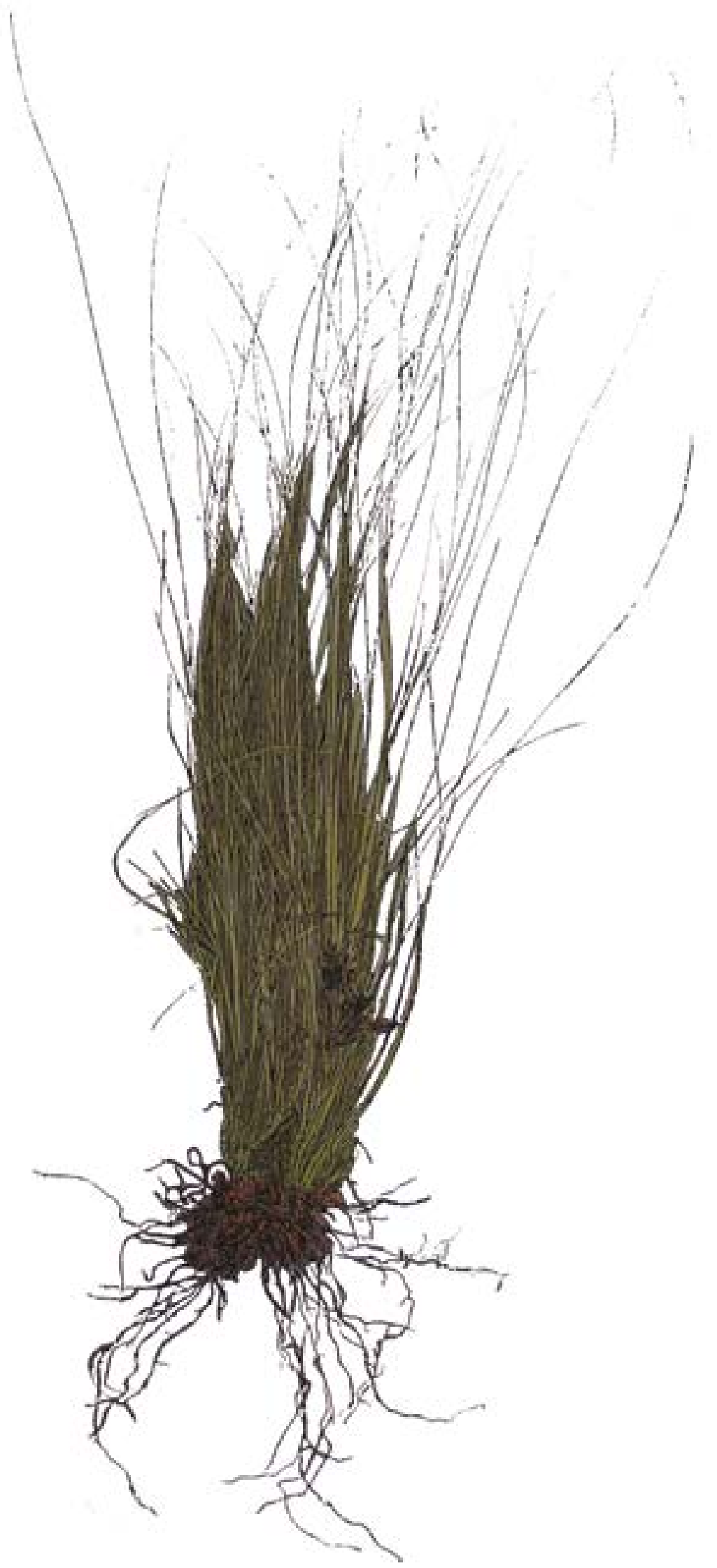

Figura 5. Gramíneas Festuca Weberbaueri Pilger Ichu blanco 


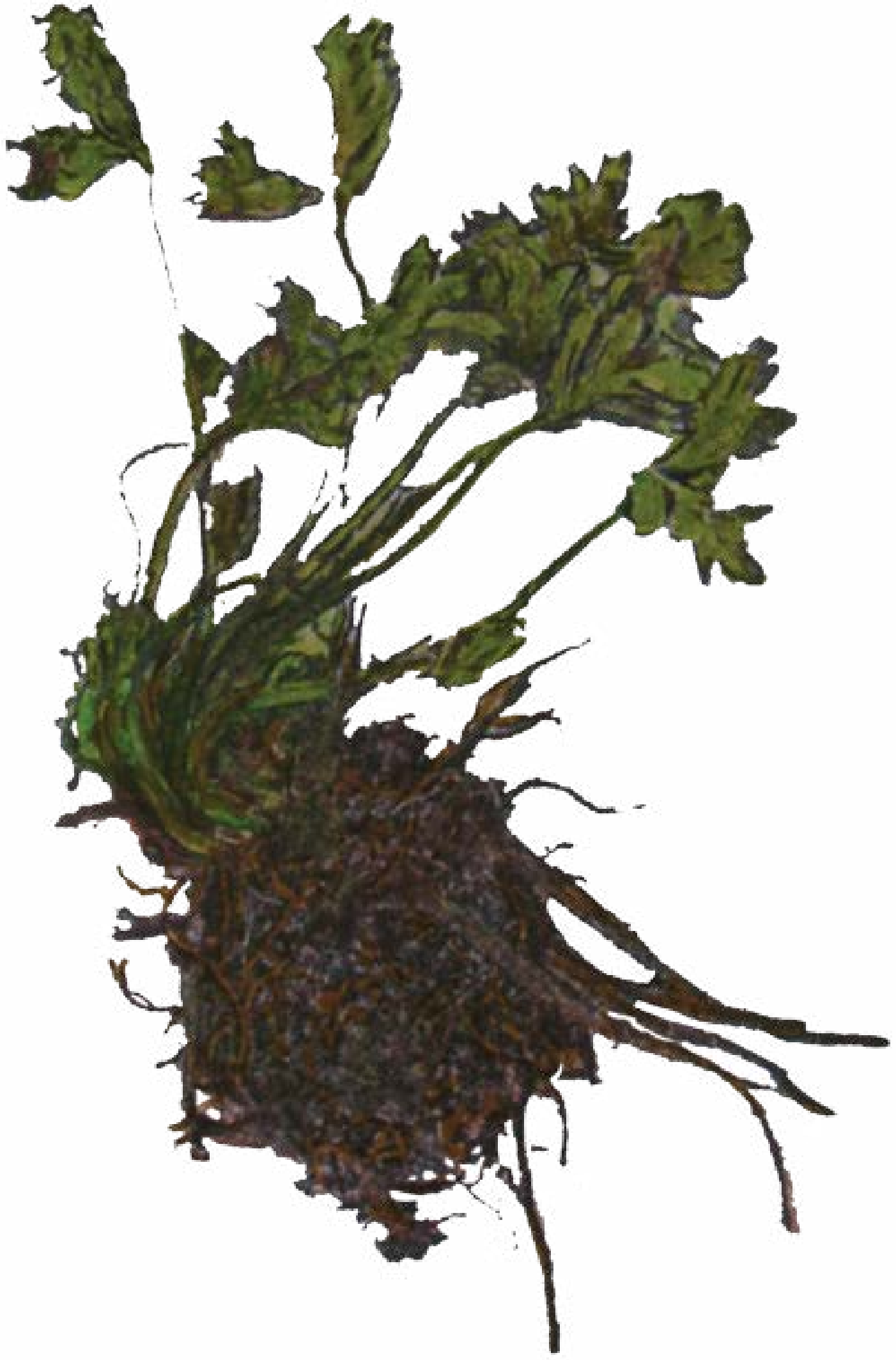

Figura 6. Geraniaceas

Geranuim patagonicum Hooker F. 